\section{Surgical outcome of single-staged three horizontal muscles squint surgery for extra-large angle exotropia}

\begin{abstract}
Purpose To report the surgical outcomes of 24 patients undergoing single-staged three horizontal muscles squint surgery for extra-large angle exotropia.

Patients and methods Prospective case series of 24 consecutive patients with primary exotropia $>60$ prism diopters (PD) at distant and underwent single-staged three horizontal muscles squint surgery from 2003 to 2006. Surgery consisted of bilateral lateral rectus recession of $9.0 \mathrm{~mm}$ for $50 \mathrm{PD}$ exotropic correction. For every 5 PD remaining angle exceeding $50 \mathrm{PD}$, we additionally performed $1.0 \mathrm{~mm}$ of unilateral medial rectus resection. The mean follow-up period was $\mathbf{1 5 . 8}$ months (range 6.0-38.0 months; SD 9.5 months).

Results The mean age at surgery was 31.2 years old (range 7-78 years old, SD 18.2 years old). The mean distant preoperative deviation was 71.3 PD (range 60-85 PD, SD 7.7 PD). No limitation of eye movement or diplopia was found. The success rate was higher in the intermittent group (88.2\%) than the constant group $(42.9 \%)(P=0.02)$ and in cases with preoperative deviation of $<80 \mathrm{PD}(84.2 \%)$ compared with those with deviation $\geqslant 80$ PD (40.0\%) $(P=0.042)$.

Conclusion Measurement of preoperative deviation and the surgery for extra-large angle exotropia made management of this condition difficult. Single-staged three horizontal muscles squint surgery can be one of the options. Further researches on management of exotropia of $>80$ PD are warranted. Eye (2010) 24, 1171-1176; doi:10.1038/eye.2010.5; published online 5 February 2010
\end{abstract}

Keywords: large angle exotropia; single staged; strabismus surgery

FHS Lau', DSP Fan², WWK Yip ${ }^{1}$, CBO Yu ${ }^{1}$ and DSC Lam²

\section{Introduction}

Large angle exotropias have great impact on patients' lives. Scatterfield et al ${ }^{1}$ described the negative impacts on strabismic patients including negative self-images, poor interpersonal relationships, school performance, and job security. A person with strabismus is considered by others to be inferior in many of the personality characteristics. ${ }^{2}$ The correction of strabismus in adults has been shown to improve the quality of psychosocial functioning for the majority of adults. ${ }^{3}$ Moreover, strabismus surgery can also realign the visual axes to eliminate diplopia and improvement of stereopsis. ${ }^{4}$ Morris et al ${ }^{5}$ and Kushner and Morton ${ }^{6}$ have found that even in adults with long-standing strabismus, they may have restoration of binocular vision after their eyes are aligned.

However, there are limited studies on the outcome of surgical management for extra-large angle exotropia. Debate still exists to what would be the best treatment. Some advocate bilateral lateral rectus recession as it is a quicker and simpler procedure and leaves the medial rectus untouched for subsequent surgery. ${ }^{7}$ Others have suggested a simultaneous three or four muscles surgery. ${ }^{8-10}$ Moreover, reference tables are mainly designed for two muscles surgery, the amount of deduction after two muscles surgery is still controversial. The few reports addressing the surgical outcomes of extra-large angle exotropia diversified in their population and the surgical method. The definition of large angle varied with some recruiting cases of $>35.0$ prism diopters (PD) only. ${ }^{11}$ The aetiologies of the cases included primary, consecutive and trauma cases. ${ }^{12}$

Individualizing the surgery by adjustable sutures may theoretically improve the surgical
${ }^{1}$ Hong Kong Eye Hospital, Kowloon, Hong Kong, People's Republic of China

${ }^{2}$ Department of Ophthalmology and Visual Sciences, The Chinese University of Hong Kong, Hong Kong Eye Hospital, Kowloon, Hong Kong, People's Republic of China

Correspondence: DSP Fan, Department of

Ophthalmology and Visual Sciences,

The Chinese University of Hong Kong, University Eye Center, 3/F, Hong Kong Eye Hospital, 147K,

Argyle Street,

Kowloon, Hong Kong, People's Republic of China. Tel: + 8522762 3150; Fax: + 85227159490 . E-mail:dorothyfan@ cuhk.edu.hk

Received: 29 May 2009 Accepted in revised form: 21 November 2009 Published online: 5 February 2010 
outcome. However, not all the patients are suitable candidates for adjustable sutures. Here, we report the surgical results of one-staged three horizontal muscles surgery for primary large angle exotropia.

\section{Materials and methods}

A prospective study was conducted on all consecutive patients with primary extra-large angle primary exotropia undergoing strabismus surgery at Hong Kong Eye Hospital from June 2003 to December 2006.

Patients with primary large angle intermittent or constant exotropia with angles $>60$ PD at distant were included. We excluded all paralytic squint, cases with history of earlier strabismus surgery, coexisting ocular disease apart from refractive error and amblyopia, restricted eye movements, or strabismus with a secondary cause. Ethics Committee approval was taken.

All patients underwent a complete ophthalmologic examination before and after the operation. The age at operation, gender, visual acuity, preoperative near and distant deviation, details of surgery, surgical complication, postoperative angle measurement, and length of follow-up were recorded. The cases were seen 1 week before the operation. They were reassessed at postoperative Day 1, Week 2, Month 1, Month 3, and then every 3 months after the operations. All the cases were followed up for at least 6 months postoperatively.

Preoperative and postoperative deviations with near $(33 \mathrm{~cm})$ and distant target $(6 \mathrm{~m})$ were measured with alternate prism cover test using loose prisms. The preoperative and postoperative sensory statuses were determined in all patients by Frisby Stereotest (Clement Clarke International Ltd, 15 Wigmore Street, London, W1H 9LA, UK).

All the operations were performed under general anaesthesia by two experienced surgeons. Both muscle recession and resection were performed with limbal approach with direct scleral fixation sutures using 6 o'vicryl (6-0 S-24 8 mm $\frac{1}{4}$ C Spatula, Ethicon Coated Vicryl, polyglactin 910, Johnson \& Johnson, Belgium). Conjunctival wounds were closed by 8 o'vicryl suture (8-0 TG140-8 6.5 mm 3/8c Spatula, Ethicon Coated Vicryl, polyglactin 910 , Johnson \& Johnson). All muscle recession measurements were measured from the muscle insertion. We adopted a surgical approach with minimal medial rectus resection. Maximal bilateral lateral rectus recessions of $9 \mathrm{~mm}$ were performed aiming for a correction of 50 PD. Any exotropia exceeding the 50 PD would be corrected by unilateral medial rectus resection on the non-dominant eye. We used an approach with $1 \mathrm{~mm}$ unilateral medial rectus resection for every 5 PD of exotropia after the bilateral lateral rectus recessions. An upwards transposition of the lateral rectus of one half tendon width was performed for patients with $\mathrm{V}$ pattern defined as angle in elevation exceeded that in depression by 15 prism dioptres. ${ }^{13}$

Successful motor alignment was defined as esodeviation between 1 and 10 PD, orthotropia or exodeviation between 1 and 10 PD during the most recent follow-up, at least 6 months postoperatively. ${ }^{14}$ A stereoacuity of $\leqslant 55 \mathrm{~s}$ of arc was considered as bifixation. A stereoacuity of $<600 \mathrm{~s}$, but $>55$ s of arc was considered as gross stereopsis. ${ }^{15}$

SPSS 11.5 for Windows statistical software was used in the analyses. $\chi^{2}$ tests were used to assess association between the variables observed. A $P$-value of $<0.05$ was considered as statistically significant.

We certify that all applicable institutional and governmental regulations concerning the ethical use of human volunteers/animals were followed during this research.

\section{Results}

There were 24 patients: 15 males and 9 females. The mean age at operation was 31.2 years. (range $7-78$ years; SD 18.2 years). Seven (29.2\%) of them had constant exotropia and $17(70.8 \%)$ had intermittent exotropia. The mean age of operation for patients in the constant exotropia group was 34.9 years and that for the intermittent exotropia group, 29.6 years $(P=0.50)$. Two $(8.3 \%)$ of the patients had anisometropic amblyopia with Snellen acuity 6/12 in the worse eye. All the remaining patients were having bilateral visual acuities equal to or greater than Snellen acuities of 6/9. The overall mean preoperative near deviation was 71.5 PD (range 60-85 PD, SD 7.7 PD) and mean preoperative distant deviation was 71.3 PD (range 60-85 PD, SD 7.7 PD). Patients in the constant exotropia group (mean 76.4 PD, range 65-85 PD, SD 9.0 PD) had a larger preoperative near deviation than the intermittent group (mean 69.4 PD, range 60-80 PD, SD $6.3 \mathrm{PD})(P=0.04)$. The mean preoperative distant deviation for the constant exotropia group was 74.3 PD (range 55-85 PD, SD 9.8 PD) and the mean deviation for the intermittent exotropia group was 69.4 PD (range 60-85 PD, SD 6.6 PD) $(P=0.166)$. Eight patients $(33.3 \%)$ were having $\mathrm{V}$ pattern, defined as above.

All patients underwent $9.0 \mathrm{~mm}$ bilateral lateral rectus recessions and unilateral medial rectus resection, according to the surgical plan described. The mean medial rectus resection performed was $4.2 \mathrm{~mm}$ (range 2.0-7.0 mm, SD $1.4 \mathrm{~mm}$ ). The mean follow-up period was 15.8 months (range 6.0-38.0 months, SD 9.5 months). No significant observable postoperative abduction deficit was noted in all the patients. One of the patients developed a conjunctival cyst postoperatively, which subside spontaneously. There were no other 
postoperative complications. The overall mean angle of postoperative exodeviation at the last follow-up for near was 10.9 PD (range 0-45 PD, SD 13.6 PD), whereas for distance was 9.9 PD (range 0-45 PD, SD 14.2 PD). For the constant exotropia group, the mean angle of postoperative exodeviation for near was 19.1 PD (range 0-40 PD, SD 17.3 PD), whereas for distance was 18.7 PD (range 0-40 PD, SD 18.8 PD). For the intermittent exotropia group, the mean angle of postoperative exodeviation for near was 7.5 PD (range 0-45 PD, SD 10.6 PD), whereas for distance was 6.2 PD (range 0-45 PD, SD 10.5 PD). Figure $1 \mathrm{a}$ and $\mathrm{b}$ shows the correction achieved (ie the difference between the pre- and postoperative
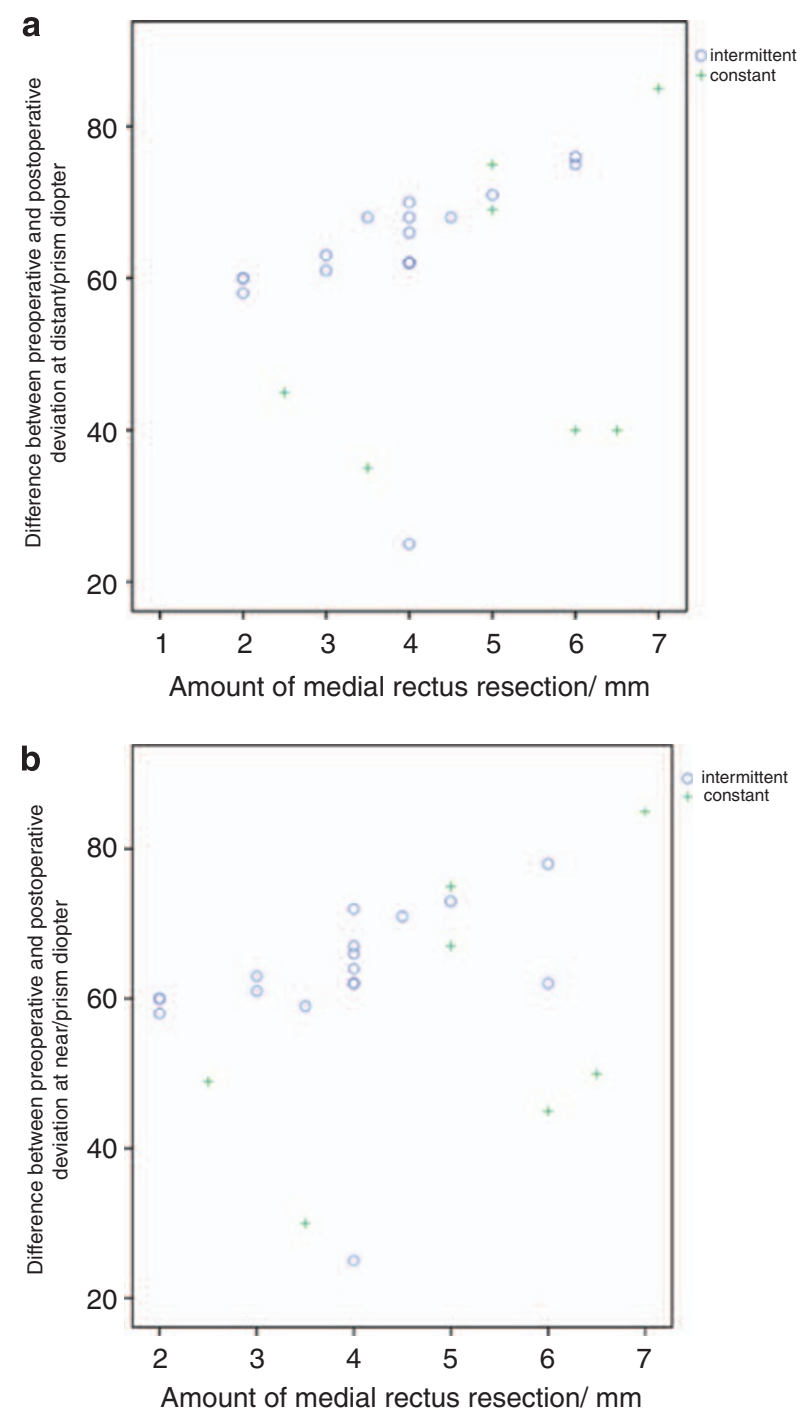

Figure 1 (a) Correction achieved (ie difference between preand postoperative angle of deviation at distant) against amount of medial rectus recession performed. (b) Correction achieved (ie difference between pre- and postoperative angle of deviation at near) against amount of medial rectus recession performed. angles) against the amount of medial rectus resection performed in the two groups of patients.

At the last follow-up, 18 of the 24 cases $(75.0 \%)$ were successfully aligned according to the criteria described above. The success rate was higher in the intermittent group $(88.2 \%)$ than the constant group $(42.9 \%)(P=0.02)$. The remaining six cases had residual exotropia or exophoria. None of the cases had overcorrection. The mean time from surgery to $>10$ PD of residual deviation was 1.7 months postoperatively (range 1.0-3.0 months, SD 1.0 month). For these patients, the mean residual postoperative angle for near was 31.5 PD (range 16.0-45.0 PD, SD 11.8 PD), and angle for distance was 30.0 PD (range 10.0-45.0 PD, SD 15.8 PD). Three out of these six patients (patient No. $5,8,19$ ) required a second operation for residual exotropia (range 40-45 PD, SD 2.9 PD). Two of them had bilateral medical rectus resections and one had unilateral medial rectus resection. All the second operations were surgically successful by the above criteria. The other three patients had good control over their residual exodeviations (range 10-35 PD, SD 14.4 PD) and they were satisfied with their cosmetic appearances, so they opted for conservative treatment. For the intermittent exotropia group, preopreatively, three patients $(17.6 \%)$ in the intermittent exotropia group achieved stereoacuity of $<55 \mathrm{~s}$ of arc, most of them $(82.4 \%)$ only achieved gross stereopsis by the criteria described above. After surgery, 11 patients (64.7\%) had achieved stereoacuity of $<55 \mathrm{~s}$. None of the patients were having postoperative diplopia at primary or side gazes. For the constant exotropia group, none of the patients have shown binocular function pre- and postoperatively. Out of the four patients who failed in the constant exotropia group, two had a second operation. All of them were satisfied with their cosmetic appearances at the latest follow-up.

For those 19 patients with preoperative distant deviation of $<80$ PD, a success rate of $84.2 \%$ was achieved, comparing with a $40 \%$ success rate in the five cases with preoperative angle of $>80.0 \mathrm{PD}(P=0.042)$.

\section{Discussion}

The surgical management of primary extra-large angle intermittent exotropia has been challenging. There is now no common consensus as to what would be the best procedure. The eligibility criteria were different in different studies. Different groups of strabismologists may also choose to perform different types of operations involving two, three, or four muscles.

Bilateral lateral recti recession was advocated by some surgeons. ${ }^{7,11,14}$ The two muscle surgery was less invasive compared with 3 or 4 muscle surgeries. However, the success rate varied from 40 to $70 \%$. If large angle 
recession was performed, it may be associated with abduction limitation. ${ }^{7}$ Owens et al ${ }^{16}$ performed monocular recession and resection, augmented with intraoperative botulinum toxin for large angle exotropia in the amblyopic eyes. However, large unilateral resection and recession procedure may produce asymmetrical abduction deficits. Velez ${ }^{17}$ reported a success rate of $40.0 \%$ by performing a unilateral recession and resection in amblyopic eyes for exotropia > 50 PD. In Berland et al series ${ }^{14}$ of 30 patients with exotropia measuring from 35 to 65 PD, the success rate was $43.0 \%$ with large bilateral lateral rectus recessions. LiverRallatos et al $^{11}$ described large angle exodeviations as deviation $>35$ PD in patients with sensory, congenital, or consecutive exotropia. Only 11 out of the 63 patients were having preoperative deviations of $>50 \mathrm{PD}$ and none of their patients had a deviation $>75$ PD. Schwartz and Calhoun ${ }^{7}$ reported a success rate of $77.0 \%$ with large two muscles recession procedure ranging from 8.0 to $14.0 \mathrm{~mm}$ in a group with mean deviation of exotropia of 58.7 PD. They used a different successful criteria of within 15 PD of orthophoria. There were postoperative abduction deficits because of the large amount of recession.

Some strabismologists would operate on four muscles to try to correct the exotropia in a single surgery. Guibor, Jampolsky, and Berke ${ }^{8-10}$ recommended operating on four horizontal muscles for very large angle exotropia. Azar ${ }^{18}$ performed bilateral lateral recessions of $10.0 \mathrm{~mm}$ with bilateral medial rectus resection of $8.0 \mathrm{~mm}$ in 29 patients with exotropia of $>70.0$ PD. Limitation of abduction was noted because of the tethering effect by the medial rectus resection. Currie et $a l^{12}$ studied 26 patients with large angle exotropia with mean near deviation of 58 PD. They included both primary and consecutive cases. They received two, three, or four muscles surgery with adjustable sutures. Seventy-seven per cent of patients were considered as successful.

Two stages surgeries were also advised: a maximum recession of the two horizontal muscles at first stage, and then proceed with a second or third procedure for the residual deviation to improve the predictability. However, the patients required to undergo more than one operation.

To the best of our knowledge, there had been no study addressing the surgical results of one-staged three horizontal muscles surgery. We studied the effect of single-staged three horizontal muscles surgery on patient with primary exodeviation $\geqslant 60 \mathrm{PD}$. This constitutes a homogenous group of primary patients that would usually not be corrected by the conventional two muscles surgery. The mean angle of deviation of our study is 71.5 PD. Our study examined the postoperative results in the correction of extra-large angle exodeviations, aiming to improve eye alignment with the minimum number of surgical interventions. Our success rate in ocular alignment was $70.0 \%$, which was comparable with the series with adjustable sutures performed. The success rate was much higher in the intermittent group $(88.2 \%)$ than the constant group (42.9\%). The results for the constant exotropia group were less encouraging and there was a high percentage of undercorrection. Although our patients did not show any significant limitation of abduction, large angle recession may predispose to movement limitation and makes further surgery more difficult. We need to be cautious in extrapolating our results to consecutive exotropia or poorly seeing eyes with secondary divergence, as the criteria for success in these groups are different.

In our series, we performed $9.0 \mathrm{~mm}$ bilateral lateral rectus recessions for all of the patients, targeting $50 \mathrm{PD}$ of exotropia correction. We tried to formulate an easily reproducible surgical plan of $1.0 \mathrm{~mm}$ unilateral medical rectus resection for every $5 \mathrm{PD}$ after bilateral $9 \mathrm{~mm}$ lateral rectus recession aiming for $50 \mathrm{PD}$ correction for patients with exotropia (Table 1). If a patient is having a preoperative angle of $80 \mathrm{PD}$, we would perform bilateral $9.0 \mathrm{~mm}$ recessions and unilateral medial rectus resection $6.0 \mathrm{~mm}$. We have obtained promising and satisfactory surgical results according to the formula above for the intermittent exotropia group, especially for cases with $<80$ PD exotropia deviation. The success rate for these cases was $84.2 \%$. On the other hand, the rate of good ocular alignment could only be achieved in $40 \%$ of cases with a deviation of $>80$ PD. Liver-Rallatos et $a l^{11}$ also showed similar finding. They found that with bilateral lateral rectus recessions, the success rate decreased significantly in deviations above 50 PD. Further refinement in surgical technique for patients with $>80$ PD exotropia deviation was required. We have chosen $9.0 \mathrm{~mm}$ bilateral lateral rectus recessions in all of our cases, as limitation of ductions after large lateral rectus recessions have been reported. ${ }^{7}$ As suggested by LiverRallatos et $a^{11}$, the functional equator lies $4 \mathrm{~mm}$ posterior to the anatomic equator laterally, approximately $11.0 \mathrm{~mm}$ posterior to the insertion of lateral rectus in an adult. Therefore, recessions greater than this amount may decrease the arc of contact of the muscle and prevent any further benefit. None of the patients in our series had any limitation in the extraocular movements. Adjustable suture may be another option. It is particularly useful when conventional surgery is unpredictable, especially when scarring, tethering, or contracture is present. ${ }^{19}$ However, it requires patients' cooperation and would be possible only in certain groups of patients.

Surgical success may not equate patient satisfaction. Burke $e t \mathrm{ll}^{3}$ found that exotropic patterns corrected to within 16.0 PD exotropia to 20.0 PD exotropia has profound satisfactory psychosocial effects. In Currie et al 
Table 1 Surgical results of the 24 patients in our series

\begin{tabular}{|c|c|c|c|c|c|c|c|c|c|}
\hline Case & $\begin{array}{l}\text { Age } \\
\text { of OT }\end{array}$ & $\begin{array}{c}\text { PreOT } \\
\text { (near) } \\
(P D)\end{array}$ & $\begin{array}{c}\text { PreOT } \\
\text { (distance) } \\
(P D)\end{array}$ & $\begin{array}{l}\text { Bilateral } L R \\
\text { recession } \\
\quad(\mathrm{mm})\end{array}$ & $\begin{array}{c}M R \\
\text { resection } \\
(\mathrm{mm})\end{array}$ & $\begin{array}{c}\text { PostOT } \\
\text { (near) }\end{array}$ & $\begin{array}{c}\text { PostOT } \\
\text { (distance) }\end{array}$ & $\begin{array}{c}\text { Follow-up } \\
\text { duration } \\
\text { (months) }\end{array}$ & $\begin{array}{c}V \\
\text { pattern }\end{array}$ \\
\hline 1 & 39 & 65 & 65 & 9 & 3 & 4 PD exophoria & 4 PD exophoria & 18 & + \\
\hline 2 & 78 & 75 & 75 & 9 & 5 & Orthophoria & Orthophoria & 31 & - \\
\hline 3 & 19 & 60 & 60 & 9 & 2 & Orthophoria & Orthophoria & 6 & + \\
\hline 4 & 14 & 70 & 70 & 9 & 4 & 4 PD exophoria & 4 PD exophoria & 13 & - \\
\hline 5 & 34 & 70 & 70 & 9 & 4 & 45 PD exotropia & 45 PD exotropia & 25 & - \\
\hline 6 & 7 & 65 & 55 & 9 & 2.5 & 16 PD exotropia & 10 PD exotropia & 23 & - \\
\hline 7 & 41 & 75 & 70 & 9 & 4.5 & 4 PD exophoria & 2 PD exophoria & 19 & - \\
\hline 8 & 22 & 85 & 80 & 9 & 6.5 & 35 PD Exotropia & 40 PD Exotropia & 38 & - \\
\hline 9 & 28 & 70 & 70 & 9 & 4 & 6 PD exophoria & 2 PD exophoria & 17 & + \\
\hline 10 & 55 & 85 & 85 & 9 & 7 & Orthophoria & Orthophoria & 12 & - \\
\hline 11 & 9 & 65 & 70 & 9 & 3.5 & 35 PD exophoria & 35 PD exophoria & 36 & - \\
\hline 12 & 51 & 75 & 70 & 9 & 4 & 8 PD exophoria & Orthophoria & 12 & - \\
\hline 13 & 28 & 70 & 70 & 9 & 4 & 8 PD exophoria & 8 PD exophoria & 8 & + \\
\hline 14 & 21 & 65 & 70 & 9 & 3.5 & 6 PD esotropia & 2 PD exophoria & 9 & + \\
\hline 15 & 31 & 80 & 80 & 9 & 6 & 2 PD exophoria & 4 PD exophoria & 6 & - \\
\hline 16 & 19 & 60 & 60 & 9 & 2 & 2 PD exophoria & 2 PD exophoria & 6 & - \\
\hline 17 & 37 & 75 & 75 & 9 & 5 & 2 PD exophoria & 4 PD esophoria & 15 & - \\
\hline 18 & 63 & 80 & 85 & 9 & 6 & 18 PD exophoria & 10 PD exotropia & 24 & - \\
\hline 19 & 24 & 85 & 80 & 9 & 6 & 40 PD exophoria & 40 PD exotropia & 18 & - \\
\hline 20 & 37 & 65 & 65 & 9 & 3 & 2 PD exophoria & 2 PD exophoria & 12 & - \\
\hline 21 & 49 & 75 & 75 & 9 & 5 & 8 PD exotropia & 6 PD exotropia & 12 & - \\
\hline 22 & 9 & 70 & 70 & 9 & 4 & 8 PD exotropia & 8 PD exotropia & 8 & + \\
\hline 23 & 20 & 60 & 60 & 9 & 2 & Orthophoria & Orthophoria & 6 & + \\
\hline 24 & 13 & 70 & 70 & 9 & 4 & 8 PD exophoria & 8 PD exotropia & 6 & + \\
\hline
\end{tabular}

Abbreviations: LR, lateral rectus; MR, medial rectus; OT, operation; PD, prism diopters; PostOT (near), postoperative near deviation; PostOT (distant), postoperative distant deviation; PreOT (near), preoperative near deviation; PreOT (distant), preoperative distant deviation.

series $^{12}, 92.0 \%$ of patients were satisfied with the outcome compared with the $72.0 \%$ surgical success rate. Most of the patients in our series were satisfied with the surgical results. Three cases with residual deviation of $>30$ PD did not want to receive further surgeries.

In conclusion, single-staged three horizontal muscles surgery for large angle intermittent exotropia has promising results with a low reoperation rate, whereas the results for constant exotropia were less promising. It can be one of the choices for patients with extra-large angle exotropia who are not suitable for adjustable sutures.

\section{Summary}

\section{What was known before}

- There are different surgeries proposed for management of large angle exotropia. On the other hand, there was no consensus yet.

\section{What this study adds}

- Single-staged three horizontal muscles squint surgery can be one of the treatment options for large angle exotropia.

\section{Conflict of interest}

The authors declare no conflict of interest.

\section{References}

1 Scatterfield D, Keltner JL, Morrison TL. Psychosocial aspects of strabismus study. Arch Ophthalmol 1993; 111: 1100-1104.

2 Olitsky SE, Sudesh S, Graziano A, Hamblen J, Brooks SE Shaha SH. The negative psychosocial impact of strabismus in adults. J AAPOS 1999; 3(4): 209-211.

3 Burke JP, Leach CM, Davis H. Psychosocial implications of strabismus surgery in adults. J Pediatr Ophthalmol Strabismus 1997; 34: 159-164.

4 Ekdawi NS, Nusz KJ, Diehl NN, Mohney BG. Postoperative outcomes in children with intermittent exotropia from a population-based cohort. J AAPOS 2009; 13: 4-7.

5 Morris RJ, Scott WE, Dickey CF. Fusion after surgical alignment of longstanding strabismus in adults. Ophthalmology 1993; 100: 135.

6 Kushner BJ, Morton GV. Postoperative binocularity in adults with longstanding strabismus. Ophthalmology 1992; 99: 316-319.

7 Schwartz RL, Calhoun JH. Surgery of large angle exotropia. J Pediatr Ophthalmol Strabismus 1980; 17(6): 359-363.

8 Guibor G. The surgical treatment of exotropia resulting from anterior intranuclear ophthalmoplegia. AJO 1950; 33: 1840.

9 Jampolsky A. Surgical management of exotropia. AJO 1958; 45: 646.

10 Rayner JW, Jampolsky A. Management of adult patients with large angle amblyopic exotropia. Ann Ophthalmol 1973; 5(1): 95-99. 
11 Liver-Rallatos G, Gunton KB, Calhoun JH. Surgical results in large angle exotropia. J AAPOS 2002; 6(2): 77-80.

12 Currie ZI, Shipman T, Burke JP. Surgical correction of large angle exotropia in adults. Eye 2003; 17(3): 334-339.

13 Pineles SL, Rosenbaum AL, Demer JL. Decreased postoperative drift in intermittent exotropia associated with A and V patterns. J AAPOS 2009; 13: 127-131.

14 Berland JE, Wilson ME, Saunders RA. Results of large (8-9 $\mathrm{mm}$ ) bilateral lateral rectus recession for exotropia. Binocular Vis Strabismus Q 1998; 13: 97-104.

15 Wu HX, Sun J, Xia X, Xu L, Xu X. Binocular status after surgery for constant and intermittent exotropia. AJO 2006; 142(5): 822-826.
16 Owens PL, Strominger MB, Rubin PA, Veronneau-Troutman $\mathrm{S}$. Large angle exotropia corrected by intraoperative botulinum toxin $\mathrm{A}$ and monocular recession resection surgery. J AAPOS 1998; 2(3): 144-146.

17 Velez G. Surgical treatment of exotropia with poor vision. Strabismus II. Proceedings of the Fourth Meeting of the International Strabismological Association 1984, pp 263-267.

18 Azar RF. Surgical management of exotropia exceeding 70 prism diopters. Ann Ophthalmol 1971; 3(2): 159-166.

19 Morris RJ, Luff A. Adjustable sutures in squint surgery. $\mathrm{Br} \mathrm{J}$ Ophthalmol 1992; 76: 560-562. 